\title{
ACOMPAÑAMIENTO PSICOSOCIAL A ESTUDIANTES DE NIVEL UNIVERSITARIO QUE TRABAJAN CON NIÑOS Y NIÑAS JORNALERAS EN LEÓN, GTO
}

\author{
Sandra Estrada-Maldonado ${ }^{1}$ \\ Universidad de Guanajuato Campus León, México \\ Universidad Iberoamericana Campus León, México
}

\begin{abstract}
RESUMEN
En México se dan flujos migratorios internos, como el caso de las familias jornaleras que viajan desde Guerrero hacia Guanajuato. Presentamos aquí una Investigación Acción que propone un modelo de acompañamiento psicosocial desde la psicología comunitaria para el caso del programa Na'Valí que incluye campamentos de atención a la niñez, con servicios inmediatos de asistencia y resguardo mientras sus padres están trabajando en los surcos, haciendo la recolección de hortalizas. El acompañamiento tiene un doble objetivo: incidir en las condiciones emocionales del grupo de voluntariado, así como conocer de qué formas afecta a este grupo de universitarios/as el contacto abrupto con una realidad que generalmente desconocían y que suele generar mucha frustración, dada la falta de alternativas de atención o de solución.
\end{abstract}

\section{Palabras clave}

Acompañamiento psicosocial, jornaleros, intersubjetividad

\begin{abstract}
In Mexico there are internal migratory flows, as in the case of day laborers traveling from Guerrero to Guanajuato. We present here an Action Research that proposes a model of psychosocial accompaniment from community psychology's point of view in the case of $\mathrm{Na}^{\prime} \mathrm{Vali}$ program which includes child care camps, with immediate assistance and shelter services while their parents are working in the furrows, doing the collection of vegetables. This accompaniment has a double objective: to influence the emotional conditions of the volunteer group, as well as to know in what ways this group of university students is affected by the abrupt contact with a reality that they generally did not know and that usually generates a lot of frustration given the lack of alternative care or solution.
\end{abstract}

\section{Keywords}

Psychosocial support, day laborers, intersubjectivity

Correspondence about this article should be addressed to Sandra Estrada-Maldonado. Profesora de la Universidad de Guanajuato Campus León y de la Universidad Iberoamericana Campus León.E-mail: sandraestrada@ugto.mx 
PSYCHOSOCIAL SUPPORT TO UNIVERSITY-LEVEL STUDENTS WORKING WITH DAY LABORERS IN LEÓN, GTO

El problema de las migraciones internas es uno de los que resultan de la aplicación de políticas económicas y sociales que, lejos de favorecer a los sectores mayormente pauperizados en regímenes neoliberales, les van colocando en situaciones cada vez más complejas y con mayores índices de precarización. En México se dan distintos flujos migratorios internos que generalmente responden a la necesidad extrema de personas y familias de buscar alternativas de ingreso económico, ante la falta de posibilidades en sus lugares de residencia. En ese sentido, los principales mercados laborales son aquellos que requieren de la movilización de personas para tareas específicas de los ciclos de cultivo, principalmente en la temporada de cosecha y recolección, generándose así el fenómeno de las familias jornaleras que cíclicamente visitan los campos de varios estados, tradicionalmente Sinaloa y Baja California. Esto fue ya descrito por Warman y Bartra en la década de los setentas como proceso de descampesinización producto del desarrollo desigual que ha favorecido al sector industrial (como se cita en Martínez, 2016).

Sin embargo, a partir del 2011 se diversifican las zonas de llegada en el país incorporándose estados del centro como Guanajuato que se convierte en uno de los mayores receptores laborales de jornaleros y jornaleras. Son varios factores los que llevan a que pueblos indígenas encuentren la ocupación de jornaleros prácticamente como la única alternativa de supervivencia; entre otros, está la disminución o imposibilidad de la pluriactividad familiar (sobre todo la producción y venta de artesanías), el desgaste de los medios y recursos para el autoconsumo; así como el creciente despojo lo que lleva a estos sectores a quedar a merced únicamente de su fuerza de trabajo para sobrevivir (Rojas Rangel, 2017).

Específicamente nos referimos a la migración de personas provenientes del estado de Guerrero, uno de los más pobres de México. Las familias de jornaleros/as pertenecen a distintas culturas y pueblos indígenas, pero se trata principalmente de mixtecos originarios de las regiones La Montaña y Costa Chica (ambas en Guerrero). Estas familias suelen llegar al estado de Guanajuato, concretamente a los campos en la periferia de la ciudad de León en abril o mayo, quedándose hasta agosto y en ocasiones trasladándose después a otros estados como Sinaloa en lo que se ha denominado migración pendular, cuando van y vienen de sus lugares de origen a estados agricultores; o migración golondrina cuando las y los jornaleros se van trasladando a diferentes lugares según las necesidades de contratación (Rojas Rangel, 2009).

Resulta destacable en esta población el hecho de que por su condición indígena y migrante suelen ser objeto de múltiples violaciones a sus derechos humanos, lo que ha sido ya descrito por otras investigaciones (Martínez-Mendizabal, 2016; Martínez \& Guzmán, 2016; Rojas Rangel, 2017; Salazar Suárez, 2018) que al hacer un recuento de esta situación mencionan que

Las condiciones de precariedad en el traslado, en las condiciones laborales y en la estancia en León, sumadas a la pobreza extrema que padecen en sus lugares de origen por la debilidad de la estructura de oportunidades, dan como resultado la violación de todos sus derechos sociales. Por otro lado, la particular forma migratoria familiar, origina una extrema vulnerabilidad, especialmente en la salud y educación de los niños, niñas y mujeres (Martínez-Mendzabal, 2016, p.3).

A la pobreza deben sumarse la discriminación y violencia estructural que viven por ser indígenas -aproximadamente el 97\%-, siendo muchas de ellas mujeres y otro tanto niñas y niños (Tlachinollan, 2013). Bajo condiciones totalmente extralegales en la contratación y empleo, se dan situaciones como el trabajo directo en los campos de familias completas, incluyendo niñas, niños, bebés y mujeres embarazadas que realizan jornadas de hasta 10 o 12 horas sin recibir, por supuesto, hidratación ni alimentación adecuada. Para dar una idea de la magnitud podemos mencionar que en el 2014 se contabilizaron 1,047 jornaleras/os, cifra que en los dos últimos años se ha incrementado considerablemente; el año pasado se contabilizaron 218 menores de acuerdo a los registros del sistema 
para el Desarrollo Integral de la Familia (DIF) municipal (Delgado, Andrade, 2016). Cabe mencionar también que hubo 5 fallecimientos infantiles ocurridos en los campos de cultivo entre el 2012 y 2014 , los cuales pudieron ser evitados, como se muestra en la siguiente tabla elaborada con datos del Centro de Desarrollo Indígena Loyola.

\section{Cuadro 1}

Menores fallecidos en Guanajuato. Migración jornalera

\begin{tabular}{|l|l|l|l|l|}
\hline \multicolumn{2}{|l|}{ Fecha } & Edad & Comunidad de origen & Causa de defunción \\
\hline 2012 & 17 de mayo & $\begin{array}{l}10 \\
\text { meses }\end{array}$ & San Pedro el Viejo & Deshidratación \\
\hline 2013 & 7 de junio & 8 meses & Cochoapa el Grande & $\begin{array}{l}\text { Gastroenteritis y neumonía, } \\
\text { desnutrición crónica }\end{array}$ \\
\hline 2013 & 21 de julio & $\begin{array}{l}2 \text { años y } \\
6 \text { meses }\end{array}$ & $\begin{array}{l}\text { Arroyo Prieto, Cochoapa el } \\
\text { Grande }\end{array}$ & Gastroenteritis \\
\hline 2014 & 14 de mayo & 4 días & $\begin{array}{l}\text { Metlatónoc } \\
\text { Comunidad Viejo }\end{array}$ & $\begin{array}{l}\text { Choque séptico, hemorragia } \\
\text { pulmonar y neumonía. }\end{array}$ \\
\hline 2014 & 20 de junio & 4 años & \begin{tabular}{l} 
Tlacuachistlahuaca \\
\hline
\end{tabular}
\end{tabular}

Tomado de Martínez (2016), con datos obtenidos del Reporte del Centro de Desarrollo Indígena Loyola y Secretaría de Salud (2014).

El Centro de Desarrollo Indígena Loyola (CDIL), organización no gubernamental asentada en la ciudad de León, específicamente a través de su programa $\mathrm{Na}^{\prime} \mathrm{Valí}^{2}$ ha desarrollado una experiencia de trabajo que incluye campamentos de atención a niñas y niños directamente en los campos de recolección, en los que se les proporcionan servicios inmediatos de asistencia tales como apoyo alimenticio, chequeos básicos de salud y actividades lúdicas, de aprendizaje y de resguardo mientras sus padres están trabajando en los surcos, haciendo la recolección de hortalizas.

Es necesario mencionar que aparentemente el incremento en la migración jornalera al estado de Guanajuato tuvo que ver con que en esta entidad federativa los controles respecto al trabajo infantil han sido laxos en comparación con estados del norte; en palabras de las propias mujeres jornaleras: 'acá si dejan trabajar con niños'.

Además, en muchos casos, el trabajo infantil ha sido normalizado por patrones y autoridades, lo que permite entonces que quienes buscan viajar con su familia vean en este territorio mayores posibilidades que en otros estados, como afirma Martínez-Mendizabal (2016): "Por razones relacionadas con la sobrevivencia, se buscan otros lugares en donde sea más flexible la supervisión legal que les permita continuar la añeja tradición campesina de incorporar la mano de obra familiar al jornal de trabajo -la niñez incluida" (p. 8).

Todo esto contribuye a que en los campos de trabajo se inserten en los surcos niñas y niños, cuyo trabajo incluso es justificado por patrones que mencionan que su estatura favorece la recolección

${ }^{2}$ Na’Valí en lengua mixteca quiere decir campamentos de niños/as, es un programa coordinado desde el Centro de Desarrollo Indígena Loyola con apoyo de las universidades de la ciudad que cuenta con tres líneas de trabajo: Investigación, Denuncia e Intervención. 
de chile, uno de los cultivos más frecuentes. Las relaciones sociales en esta dinámica incluyen un empresario agrícola, quien posee extensiones relativamente grandes de tierra y que, a través de un caporal, contrata siempre informalmente a jornaleras/os e incluso a grupos familiares o comunidades casi completas, quienes vienen con la ilusión de ahorrar dinero para regresar a su tierra, aunque esto no siempre se logre o no sea por el tiempo que pensaban.

La jornada de trabajo suele iniciar entre las 6 y 7 de la mañana cuando pasan por ellos y les llevan en camionetas al campo indicado, trabajarán allí jornadas de 10 horas regularmente y van a recibir un pago a destajo que en los mejores casos llega semanalmente. El pago se hace en base al número de sacos o botes de chile recolectado, que suelen pesar entre 20 y 25 kilogramos, y este pago a destajo favorece el involucramiento de niñas y niños que ayudan a los adultos para llenar un mayor número de botes o costales.

Ha sido justamente esta alta presencia de niños y niñas en los campos lo que motivó originalmente al CDIL a implementar el proyecto Na'valí, que a través de la instalación de campamentos y equipos de voluntarios asiste a las familias jornaleras. Estos campamentos suelen estar sostenidos principalmente por estudiantes de nivel universitario, que son capacitados y coordinados por el CDIL, que se encarga a su vez de gestionar apoyos financieros durante todo el año.

La convocatoria para participar como voluntario/a se hace a través de las instituciones educativas y generalmente como parte de los proyectos de servicio social a los que pueden inscribirse, ya sea para sumar créditos o para cubrir requisitos de cada institución. Cabe destacar que en ningún caso se trata de una participación forzada o a la que se les obligue.

Los equipos de Na'Valí, después de recibir capacitación específica, implementan en los campos acciones en tres ejes: educativo, lúdico y nutricional. Las jornadas de trabajo suelen ser muy pesadas pues comienzan a las 8:00 am y permanecen en los campos de cultivo de 9:00 am a 2:00 pm, en este lapso se imparten actividades tanto de socialización como de aprendizaje con niñas y niños, resguardándolos además de las inclemencias del sol al que suelen estar expuestos.

Presentamos aquí la experiencia de acompañamiento psicosocial que realizamos desde el marco de la psicología social comunitaria con el equipo de voluntariado que está directamente atendiendo a las familias. El objetivo es doble, tratándose de una investigación-acción, pues por un lado nos interesó incidir en la mejora de las condiciones anímicas y emocionales del grupo de voluntariado para a su vez mejorar el trabajo en el proyecto Na'Valí. Pero al mismo tiempo buscamos también conocer de qué formas afecta a este grupo de universitarios/as el contacto abrupto con una realidad que generalmente desconocían y que suele generar mucha frustración, dada la falta de alternativas de atención o de solución. Para ello nos basamos en el concepto de intersubjetividad como herramienta tanto para la generación y encuadre del espacio de conversatorio, como para el análisis de cada encuentro semanal, pues la entendemos como una "creación e intercambio de símbolos en la vida cotidiana que reflejan una manera compartida de pensar, sentir y actuar" (Almeida, 2014, p.133).

Este trabajo se ubica en los desafíos actuales de la Psicología Comunitaria, pues nos propone acompañar a familias jornaleras que justamente están fuera de sus comunidades de origen, y que además se encuentran inmersas en nuevas relaciones sociales ligadas ahora a los procesos de explotación en el campo. Por ejemplo, quienes eran sólo vecinos o parientes en Guerrero, ahora en los campos de León pueden pasar a ser caporales, con el agravante de que se encuentran en un territorio ajeno. Esto nos lleva a seguir la invitación de Montenegro, Rodríguez y Pujol de "Pensar la comunidad en términos de conexión de diferencias en lugar de agrupaciones de comunalidades", permitiéndonos de este modo "conceptualizar los espacios en que intervenimos como intrínsecamente diaspóricos, con una tensión constante entre los espacios identitarios que nos constituyen, potencialmente diversos geográficamente y los lugares físicos que habitamos, inherentemente interseccionales" (2014, p.39).

\section{Método}


El trabajo que aquí presentamos se fue construyendo a partir de la solicitud de la coordinación del proyecto Na'Valí de abrir un espacio de contención para quienes participaban como voluntarias/os, pues el enfrentarse a las condiciones de niñas y niños les generaba en algunos casos altos índices de ansiedad o emociones que no podían manejar. Fue a raíz de esta solicitud que construimos conversatorios como espacios de diálogo y escucha, en general sobre el diseño e implementación de una propuesta de acompañamiento psicosocial, abordada desde una perspectiva de Investigación Acción Participativa (IAP) que permitiera recuperar información de manera simultánea a la intervención misma, procurando con ello generar las condiciones idóneas para que quienes participaran como voluntarios/as integraran y procesaran de manera menos disruptiva el encuentro con una realidad que hasta antes del proyecto les resultaba ajena. En este sentido retomamos el planteamiento de Martín Baró, cuando afirma que "la preocupación del científico social no debe cifrarse tanto en explicar el mundo cuanto en transformarlo" (1986, p.3),

Al asumir una perspectiva de investigación participante, retomamos los elementos propuestos por Hall cuando menciona que se trata de "una actividad integrada que combina la investigación social, la labor educacional y la acción" (como se cita en Demo, 2009, p.81) y que no se agota en un producto académico sino al contrario, busca un beneficio directo para la comunidad manteniendo en todo momento su carácter dialéctico (Demo, 2009).

Asumimos también, como lineamiento ético, el hecho de que la opción epistémica y metodológica de la IAP se basa en una ética relacional, no reificante de la otredad, y que comprende a la investigación y la intervención comunitaria como parte de un mismo proceso. Fals Borda la describe como:

Una metodología dentro de un proceso vivencial, un ciclo productivo satisfactorio de vida y trabajo en las comunidades en busca de 'poder' y no tan sólo de 'desarrollo' para los pueblos de base, un proceso que incluye simultáneamente educación de adultos, investigación científica y acción política, y en el cual se consideran el análisis crítico, el diagnóstico de situaciones y la práctica como fuentes de conocimiento. (1985, p. 125).

También es importante destacar que situamos las actividades en un nivel de acompañamiento, evitando con ello caer en la idea de intervención como una acción que resalta el rol de experto de quien investiga o interviene; por el contrario, asumimos que "El nivel de acompañamiento renuncia a las relaciones verticales y jerárquicas, muy tradicionales en la intervención psicológica; más bien nos posicionamos como testigos, facilitadores y acompañantes de alguien en su proceso" (Martínez, Estrada, 2014, p.234) buscando también movernos de la dimensión individual a la dimensión psicosocial.

En este marco de IAP, construimos conversatorios, entendiéndolos como espacios dialógicos de contención y reflexión que semanalmente proporcionaron encuentros en los que a través de la escucha activa se promovía el relato vivencial, destacando los nudos de conflicto o las emociones incómodas que pudieran surgir, tales como: coraje, culpa, enojo hacia las madres y los padres (situación que eventualmente puede llevar a la criminalización de las familias), y procuramos la resignificación de las experiencias vividas para evitar que esto pueda entorpecer de alguna manera el trabajo cotidiano que realizan en los campos agrícolas.

Elegimos metodologías participantes por su "potencial de contribuir a construir conocimientos relevantes a los problemas que le atañen y a modificar las condiciones que los suscitaron" (Wiesenfeld, 2014, p.10), pues al tratarse de un trabajo durante 3 a 4 meses es muy importante garantizar simultaneidad en los procesos de recolección de información y de transformación mediante el acompañamiento. Evidentemente el interés mayor de este proyecto es que voluntarias y voluntarios se involucren con niñas y niños, procurando relaciones horizontales que no estén obstruidas por sus 
propios prejuicios, frustraciones, etc. Es decir, buscamos que el impacto de mejorar las condiciones anímicas del equipo de voluntariado redunde al final en acompañamientos más empáticos hacia la niñez jornalera.

El presente ejercicio pretende también rescatar las interacciones en el sentido planteado por Fernández-Christlieb (como se cita en Almeida, 2014), en tanto proceso en el que se crean e intercambian significados; procurando que los encuentros cara a cara de los conversatorios trascendieran hacia mayores dimensiones de análisis e incorporaran otros significados orientándose en mayor medida hacia la potenciación de los lazos comunitarios.

En estos espacios se consideraron algunas de las dimensiones que tradicionalmente han sido reconocidas como variables que determinan el nivel de afectación en quienes trabajan en situaciones críticas (Armayones-Ruiz, 2014), es decir:

- Factores personales: edad, características de personalidad, situación social y personal, sistema de creencias, etc.

- Factores profesionales: experiencia en este tipo de trabajo, atención en situaciones de crisis, relación entre voluntarias/os, clima laboral, etc.

- Emociones presentes durante las experiencias en campo: temor, inseguridad, culpa, pena, enojo, tristeza, etc.

Los conversatorios incluyeron un total de 12 sesiones de 60 minutos con una participación de entre 8 y 20 voluntarias/os distribuidas a partir de mayo y hasta julio. En estos encuentros, la coordinadora presentaba alguna técnica o dispositivo breve que permitiera generar la discusión y a lo largo de las intervenciones se iban puntualizando y señalando aquellos aspectos que permitían orientar el trabajo hacia la potenciación intersubjetiva y mediante una ética relacional, alejándose así de la visión puramente asistencialista y o de caridad.

\section{Gráfico}

Sesión de conversatorio con jóvenes estudiantes

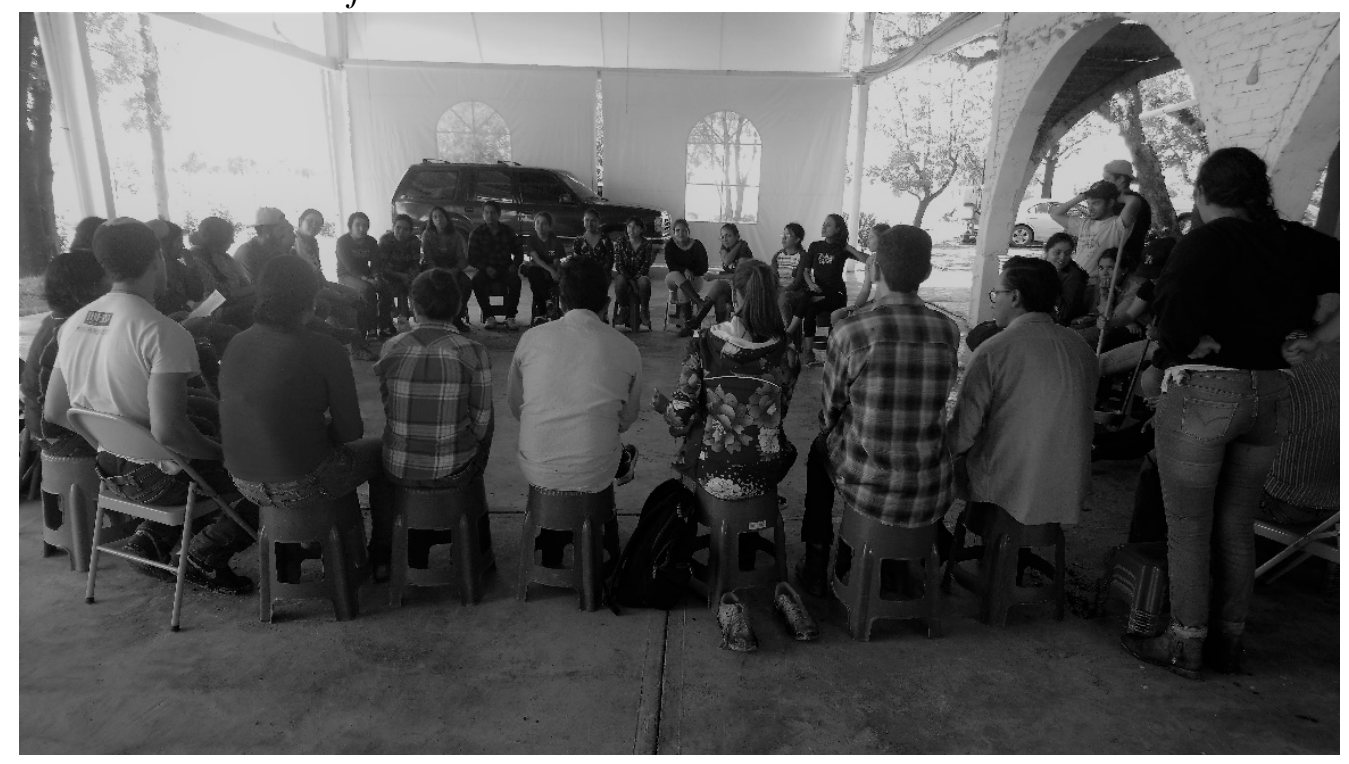

Para el análisis se contó con una observadora, quien realizaba una relatoría de cada sesión, la cual era después complementada con notas y observaciones de la facilitadora; a partir de ello se fueron decantando los ejes de trabajo que describiremos más adelante.

Atendiendo al ciclo rítmico de la Investigación-Acción que contempla observaciones, acción concreta o práctica, vuelta a la reflexión, producción de preconceptos y vuelta al comienzo (Fals Borda, 2009), emprendimos este camino buscando que cada conversatorio fuera un episodio singular y acabado que permitiera reflexionar y pensar en nuevos planteamientos para la siguiente semana, 
pero sin ser acontecimientos hilados ni formando parte de un proceso de pura observación o de pura intervención, buscamos articular estos espacios como praxis intersubjetiva y potenciadora.

Sin pretender que los conversatorios estuvieran totalmente centrados en la abreacción o debriefing como metodología, sí promovimos algunos de sus componentes como la posibilidad de "descargar la tensión emocional generada por las situaciones impactantes que provocan sufrimiento y malestar" (Santacruz, 2009, p.199), pues en varias ocasiones para los participantes resultaba muy frustrante no poder manejar situaciones de disciplina o bien momentos en los que acumulaban enojo y/o coraje hacia los padres porque no permitían que sus hijos salieran del surco al campamento, ya que querían que les ayudaran en la pizca. Esto genera en las y los estudiantes una fuerte contradicción pues se integran en el equipo con el afán de ayudar a niños y niñas, pero pocas veces se imaginan que los padres no sólo llevan a los niños al campo porque no tienen opción de dejarlo al cuidado de alguien, sino también porque de esa manera el trabajo de niños y niñas también contribuye al ingreso familiar puesto que el pago es a destajo.

Con el objetivo de evitar que esas emociones permanezcan y se conviertan en juicios constantes hacia las familias se abren los conversatorios, procurando que la experiencia personal y subjetiva sea espejeada en el grupo y que los prejuicios puedan ser al menos cuestionados y resignificados.

\section{Resultados y Discusión}

El dispositivo para el trabajo grupal, como ya se mencionó, fue el esquema de conversatorio contemplando para cada sesión algún elemento detonador de la conversación, que sin tratarse de un plan rígido permitiera rescatar los aspectos significativos o problemáticos a partir de las vivencias que referían las y los participantes que hubieran tenido lugar a lo largo de la semana.

Partiendo del registro escrito de las sesiones, observamos que existían tópicos que surgían recurrentemente incorporando la experiencia de dos ciclos: 2017 y 2018; lo cual nos llevó a pensar en posibles ejes temáticos que permitieran consolidar un modelo de acompañamiento, pues encontramos que estas insistencias estaban evidentemente ligadas a las necesidades y nudos de conflicto que se van presentando en el equipo de voluntariado.

Si bien el conversatorio debe ser lo suficientemente flexible que permita incorporar las subjetividades de cada integrante y la diferencia con la que cada uno/a va vivenciando la experiencia, también se trata de un espacio que hace visible las necesidades colectivas, y es en base a ello que encontramos los siguientes temas que no necesariamente se presentan en orden de prioridad, sino simplemente como un listado de elementos de la dimensión psicoafectiva que deben ser considerados. Presentamos a continuación algunas de las expresiones de estudiantes obtenidas de estos conversatorios y que fueron el punto de partida para reflexiones y diálogos:

- La falta de educación sexual hace que estas personas sigan teniendo hijos cuando no los pueden mantener.

- Me siento muy mal porque está lloviendo, a veces cuando los dejamos está lloviendo y los niños se quedan en el campo.

- Sabía que la experiencia iba a ser difícil por sus condiciones. pero no me imaginé que tanto.

- Me da tristeza pensar que "esa es su realidad todo el tiempo".

- Me siento frustrada porque pienso que lo que hacemos es "super insignificante".

- No puedo concentrarme por el coraje, "no se me hace justo, pienso que yo pude haber nacido ahî".

- Son ignorantes y por eso no quieren dejar que sus hijos aprendan. 
Como se puede ver, estas afirmaciones están también cargadas del racismo y clasismo tan presente en México hacia sus pueblos indígenas, como ya se ha documentado en otras investigaciones (Gall, 2004; Schmelkes 2009). Esto además se acentúa en la zona centro del país, al tratarse de poblaciones urbanas y con poca presencia de indígenas si se compara con los estados mexicanos del sur o sureste; además de que las y los estudiantes provienen de sectores de élite, ya sea medios o altos pero que definitivamente están distantes de la realidad de pobreza de las familias jornaleras.

El trabajo en los conversatorios consistió justamente en partir de alguna de estas expresiones y buscar resignificarla a partir de las vivencias del grupo, incorporando elementos que movilizaran la conciencia y permitieran una mejor problematización y desnaturalización de prejuicios.

Decidimos agrupar los tópicos en base a ejes temáticos para dar la noción de porosidad en cada uno de ellos, ya que no se trata de categorías estancas sino de áreas de trabajo que pueden incluso tener fronteras difusas entre sí.

Los siguientes son los ejes que consideramos base para un modelo de atención y acompañamiento flexible pero, a su vez, con actividades específicas que permitan detonar en cada conversatorio la reflexión y acompañamiento grupal a las inquietudes individuales en el voluntariado o viceversa.

\begin{tabular}{|c|c|}
\hline Eje temático & Descripción \\
\hline a) Prejuicios y estereotipos & $\begin{array}{l}\text { Identificación y análisis de los prejuicios y } \\
\text { estereotipos hacia la pobreza y los pueblos } \\
\text { indígenas. Más allá de la capacitación y de la } \\
\text { información que puedan tener, en la } \\
\text { experiencia cotidiana suelen aflorar los } \\
\text { prejuicios que no han sido re-elaborados, por lo } \\
\text { que en cada conversatorio hay que prestar } \\
\text { atención a ello. }\end{array}$ \\
\hline b) Dimensión emocional & $\begin{array}{l}\text { Reconocimiento de las emociones y } \\
\text { desmitificación de su carácter negativo o } \\
\text { positivo, para permitir con ello la expresión } \\
\text { asertiva, así como la tolerancia en el equipo. }\end{array}$ \\
\hline c) Empatía & $\begin{array}{l}\text { Intencionar la empatía, tanto hacia } \\
\text { compañeras/os como hacia las propias familias } \\
\text { jornaleras, de manera que se reduzcan los } \\
\text { juicios sobre las acciones de los demás. }\end{array}$ \\
\hline d)Solidaridad & $\begin{array}{l}\text { Enfatizar el carácter de atención solidaria } \\
\text { versus ayuda caritativa, cuidando promover } \\
\text { relaciones e interacciones horizontales sobre lo } \\
\text { que tenemos en común con los jornaleros más } \\
\text { que sobre las diferencias. }\end{array}$ \\
\hline e) Dignidad & $\begin{array}{l}\text { Destacar la relevancia de mantener a lo largo } \\
\text { del trabajo la dignidad, tanto de quienes } \\
\text { reciben atención como de quienes la brindan. }\end{array}$ \\
\hline f) Prevención de efectos secundarios & $\begin{array}{l}\text { Cuidar que los posibles sentimientos de culpa, } \\
\text { impotencia, frustración o enojo no impidan el } \\
\text { trabajo cotidiano en las acciones del proyecto y } \\
\text { que tampoco afecten negativamente en cada } \\
\text { voluntario/a. }\end{array}$ \\
\hline
\end{tabular}


Reconocer estos ejes nos ha sido de gran utilidad, pues permitió que quien facilitaba los conversatorios pudiera ir acomodando el material discursivo emergente en el grupo en torno a alguno de ellos, contribuyendo así a un proceso direccionado a los objetivos planteados. De otro modo se correría el riesgo de que pareciera un grupo terapéutico o de improvisación cuando no era así, ya que se buscaba también una transferencia técnica en cuanto al manejo emocional y a la comprensión de la complejidad del problema de la migración indígena. Decimos esto al considerar que lo que hemos visto en estos espacios es que al desconocer la realidad; estudiantes y voluntarios tienden a emitir juicios más severos y en general a tener una perspectiva simplista de la problemática, asumiendo que las causas se reducen a la esfera familiar o incluso individual. En este sentido, la mirada desde lo psicocomunitario aporta en buena medida la posibilidad de ampliar y complejizar la perspectiva de las y los estudiantes voluntarios.

Una de las ventajas obtenidas con estos conversatorios es que se logró incorporar las dimensiones histórica y económica de los procesos migratorios, no a gran profundidad pero sí al menos lo suficiente para evitar caer en la psicologización del fenómeno, haciendo reducciones convenientes y contribuyendo también a dimensionar los alcances del proyecto, pues las condiciones de niños y niñas son tan adversas y dolorosas que generan en los estudiantes sentimientos de culpa y de mucha frustración e impotencia.

Del mismo modo fue muy importante hacerle ver a la institución (el CDIL) la importancia de contar con este espacio de contención psicológica, pues eventualmente la frustración y enojo que puede llegar a acumularse hace que las y los voluntarios se vuelvan mucho más duros hacia las familias, lo cual obstruye el acompañamiento y el trabajo al interior de los equipos.

\section{Conclusiones}

La presencia de niñas y niños en los campos de recolección evidencia terribles condiciones de vida en nuestro país, pues la precariedad endémica desde sus lugares de origen y el desamparo de los campos agrícolas hacen que padezcan las tradicionales enfermedades de la pobreza: desnutrición crónica, dermatosis, conjuntivitis, enfermedades gastrointestinales, infecciones recurrentes de vías respiratorias (Martínez-Mendizabal, 2016). Esto se suma al racismo velado o explícito de una sociedad conservadora como la guanajuatense y a la indiferencia e incompetencia de instancias gubernamentales, que también hacen lo propio para que esta realidad sea permanentemente invisibilizada.

Todo esto evidencia las grandes contradicciones en una sociedad que no fortalece el vínculo de transmisión intergeneracional que sostiene singular y socialmente para abrir o iniciar otro camino, sino que por el contrario ubica a niñas y niños en el campo de la miseria, como infancia-resto haciéndoles víctimas "de un doble despojo: del lenguaje y de la materialidad del ser en el mundo" (Lenta, Zaldúa, 2016, p.258). Esta incongruencia es a su vez develada por el equipo de jóvenes estudiantes que hacen su voluntariado o servicio social en el proyecto Na'valí, dejándoles en un lugar de alta vulnerabilidad emocional pues no son todavía parte formal de ese mundo adulto que carga con la mayor responsabilidad hacia la niñez, pero sí están en el umbral de la vida profesional, lo cual incrementa de manera muy fuerte la frustración y la culpa después de haber compartido las mañanas en los surcos con estas familias jornaleras y pensar en la vuelta a su vida cotidiana.

En este sentido, los conversatorios propuestos para potenciar la intersubjetividad han abonado a la ruptura de significados producto de situaciones enajenantes para orientarse hacia nuevas formas de intersubjetividades (Almeida, 2014), fortaleciendo el tejido social y haciendo de esta experiencia no sólo un quiebre personal sino también un ejercicio de resignificación colectiva.

La psicología comunitaria suele asociarse al trabajo exclusivo con sectores empobrecidos y vulnerables, sin embargo, en este caso presentamos un tipo de intervención que si bien apunta a las niñas y niños jornaleros se desarrolla mediante el acompañamiento psicosocial a los grupos de universitarios/as voluntarios/as. Se consolida el conversatorio grupal como dispositivo en dos 
sentidos, tanto el cognitivo que permite problematizar y resignificar las preconcepciones de cada uno/a; como en el emocional que a su vez facilita el reconocimiento y manejo de las emociones al enfrentarse a situaciones críticas y convivir con poblaciones tan vulneradas. Todo ello desde una perspectiva psico comunitaria que permitió desentrañar los aspectos complejos e histórico sociales para que su comprensión se tradujera finalmente en mejores prácticas de las y los voluntarios hacia la niñez jornalera e indígena. 


\section{Referencias}

Almeida-Acosta E.; Sánchez-Díaz de Rivera, M.E. (2014). Comunidad: interacción, conflicto y utopía.Puebla, México: Universidad Iberoamericana

Armayones-Ruiz, M. (2014). Técnicas de apoyo psicológico y social en situaciones de crisis: cómo desarrollar las habilidades adecuadas ante situaciones de emergencia. Madrid :Ideaspropias Editorial SL.

Delgado, D.; Andrade, J. (15 de mayo de 2016). Reclaman apoyo nulo a jornaleros. Periódico AM, sección local. León, Gto. Disponible en https://www.am.com.mx/2016/06/14/leon/local/reclaman-nuloapoyo-a-jornaleros-291522

Demo, P. (2009). Investigación participante: mito y realidad. Buenos Aires: Lumen Humanitas.

Fals Borda, O. (1985). Conocimiento y poder popular. Lecciones con campesinos de Nicaragua, México y Colombia. Bogotá: Punta de Lanza, Siglo XXI.

Fals Borda, O. (2009). Cómo investigar la realidad para transformarla, en Una sociología sentipensante para América Latina. Bogotá, Colombia: CLACSO-Siglo del Hombre.

Gall, O. (2004). Identidad, exclusión y racismo: reflexiones teóricas y sobre México. Revista mexicana de sociología, 66(2), 221-259.

Lenta, M.; Zaldúa, G. (2016). Niñez, adolescencia y derechos humanos. En Zaldúa, G (coord.) Intervenciones en Psicología Social Comunitaria. Buenos Aires: Teseo.

Martín Baró (1986). Hacia una psicología de la liberación. Boletín de Psicología, (22), 219-231.

Martínez-Mendizabal, D. (2016). Jornaleros mixtecos migrantes en la zona agrícola de León, Guanajuato: El holograma del régimen de bienestar en México. León, México: Universidad Iberoamericana León.

Martínez Mendizábal, D., \& Estrada Maldonado, S. (2014). Propuesta de acompañamiento psicosocial con familias migrantes. Sinéctica, (43), 01-14. Disponible en http://www.scielo.org.mx/scielo.php?pid=S1665109X2014000200010\&script=sci_arttext\&tlng=pt

Martínez, D., \& Guzmán, P. (2016). Familias mixtecas jornaleras: un éxodo dramático. León, México: Universidad Iberoamericana León y Centro de Desarrollo Indígena Loyola.

Rojas Rangel, T. (2009). La crisis del sector rural y el coste migratorio en México. Iberoforum. Revista de Ciencias Sociales de la Universidad Iberoamericana, 4(8). Disponible en https://www.redalyc.org/html/2110/211014822002/

Rojas Rangel, T. (2017). Migración rural jornalera en México: la circularidad de la pobreza. Iberoforum. Revista de Ciencias Sociales de la Universidad Iberoamericana, XII (23), 1-35. Disponible en https://www.redalyc.org/html/2110/211053027001/

Salazar, M. N. (2018). Mercado de fuerza de trabajo rural en México. Pegada-a Revista da Geografia do Trabalho, 19(1). Disponible en http://revista.fct.unesp.br/index.php/pegada/article/view/5747

Santacruz Escudero, J. M. (2008). Una revisión acerca del debriefing como intervención en crisis y para la prevención del TEPT (trastorno de estrés postraumático). Revista colombiana de psiquiatría, 37(1). Disponible en https://www.redalyc.org/html/806/80615420016/

Schmelkes del Valle, S. (2009). Interculturalidad, democracia y formación valoral en México. Revista electrónica de investigación educativa, 11(2), 1-10. Disponible en http://www.scielo.org.mx/scielo.php?pid=S1607-40412009000200004\&script=sci_arttext

Tlachinollan (2013). La montaña de Guerrero: tierra de mujeres migrantes. México: Tlachinollan. Wiesenfeld, E. (2014). La Psicología Social Comunitaria en América Latina: ¿Consolidación o 
crisis? Psicoperspectivas, 13(2), 6-18. Disponible en https://scielo.conicyt.cl/scielo.php?pid=S0718-

$69242014000200002 \&$ script=sci_arttext\&tlng=en

Received: $12 / 18 / 2018$

Accepted: 06/14/2019 